\title{
Diagnostic and prognostic value of CEP55 in clear cell renal cell carcinoma as determined by bioinformatics analysis
}

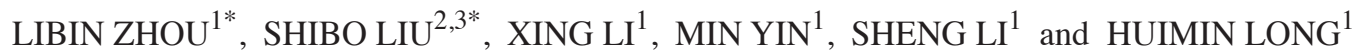 \\ ${ }^{1}$ Department of Urology, Ningbo Medical Centre Lihuili Hospital, Ningbo, Zhejiang 315040; \\ ${ }^{2}$ Department of Urology, Cancer Hospital of China Medical University; ${ }^{3}$ Department of Urology, \\ Liaoning Cancer Hospital and Institute, Shenyang, Liaoning 110042, P.R. China
}

Received October 11, 2018; Accepted March 1, 2019

DOI: $10.3892 / \mathrm{mmr} .2019 .10042$

\begin{abstract}
Clear cell renal cell carcinoma (ccRCC) is one of the most common types of malignant adult kidney tumor. Tumor recurrence and metastasis is the primary cause of cancer-associated mortality in patients with ccRCC. Therefore, identification of efficient diagnostic and prognostic molecular markers may improve survival times. The GSE46699, GSE36895, GSE53000 and GSE53757 gene datasets were downloaded from the Gene Expression Omnibus database and contained $196 \mathrm{ccRCC}$ samples and 164 adjacent normal kidney samples. Bioinformatics analysis was used to integrate the four microarray datasets to identify and analyze differentially expressed genes. Functional analysis revealed that there were 12 genes associated with cancer, based on the tumor-associated gene database. Erb-B2 receptor tyrosine kinase 4, centrosomal protein 55 (CEP55) and vascular endothelial growth factor A are oncogenes, all of which were associated with tumor stage, whereas only CEP55 was significantly associated with survival time as determined by Gene Expression Profiling Interactive Analysis. The mRNA expression levels of CEP55 in ccRCC
\end{abstract}

Correspondence to: Dr Huimin Long, Department of Urology, Ningbo Medical Centre Lihuili Hospital, 57 Xingning Road, Ningbo, Zhejiang 315040, P.R. China

E-mail: longhuiming@vip.sina.com

*Contributed equally

Abbreviations: CEP55, centrosomal protein 55; ccRCC, clear cell renal cell carcinoma; GEO, Gene Expression Omnibus; DEGs, differentially expressed genes; ERBB4, Erb-B2 receptor tyrosine kinase 4; VEGFA, vascular endothelial growth factor A; GEPIA, Gene Expression Profiling Interactive Analysis; TCGA, The Cancer Genome Atlas; ROC, receiver operating characteristic; AUC, area under the curve; RRA, Robust Rank Aggregation; TAG, tumor-associated gene; CI, confidence interval; KEGG, Kyoto Encyclopedia of Genes and Genomes; GSEA, gene set enrichment analysis

Key words: clear cell renal cell carcinoma, bioinformatics, diagnosis, prognosis, centrosomal protein 55 samples were significantly higher than those observed in adjacent normal kidney tissues based on The Cancer Genome Atlas data and reverse transcription-polymerase chain reaction results. The receiver operating characteristic curve analysis revealed that CEP55 may be considered a diagnostic biomarker for ccRCC with an area under the curve of $>0.85$ in the training and validation sets. High CEP55 expression was strongly associated with sex, histological grade, stage, $\mathrm{T}$ classification, $\mathrm{N}$ classification and $\mathrm{M}$ classification. Univariate and multivariate Cox proportional hazards analyses demonstrated that CEP55 expression was an independent risk factor for poor prognosis. In addition, gene set enrichment analysis indicated that high CEP55 expression was associated with immunization, cell adhesion, inflammation, the Janus kinase/signal transducer and activator of transcription signaling pathway and cell proliferation. In conclusion, CEP55 was increased in ccRCC samples, and may be considered a potential diagnostic and prognostic biomarker for ccRCC.

\section{Introduction}

Renal cell carcinoma (RCC) is one of the 10 most frequent types of cancer in women and men, accounting for $\leq 3 \%$ of all adult cancers. Types of RCC include clear cell (70\%), papillary $(10-15 \%)$, and chromophobe $(5 \%)(1,2)$. Clear cell RCC (ccRCC) is the main pathological type of primary renal tumor, which is associated with increased incidence, and high morbidity and mortality (3). Through the use of abdominal imaging, patients with ccRCC are being more frequently identified (4); however, this type of cancer is insensitive to radiotherapy and chemotherapy, and the 5-year survival rate of advanced stage patients following diagnosis is $<10 \%$ (5). Several multi-center international studies have demonstrated that local or distant recurrence occurs in $20-40 \%$ of patients with localized tumors following nephrectomy (6). Therefore, uncovering the underlying molecular mechanisms, and identifying effective diagnostic and prognostic molecular markers may help explore novel drug targets to control the proliferation, metastasis and drug resistance of ccRCC.

With the development of improved computer technology, high-throughput platforms have been widely applied for gene expression analysis. Microarray analysis and next-generation sequencing are increasingly utilized as essential tools in the 
field of medical oncology, with numerous clinical applications, including molecular classification of cancer, molecular diagnosis, prognosis prediction, patient stratification, tumor response prediction and novel drug target discovery $(7,8)$. Furthermore, various tumor-associated databases have been established, including the Gene Expression Omnibus (GEO) and The Cancer Genome Atlas (TCGA). The GEO is an international public repository that contains a number of high-throughput microarray and next-generation sequencing datasets (9). TCGA is a public database that includes 33 cancer types with matched clinical data, mRNA data and microRNA data $(10,11)$. The methods associated with bioinformatics analysis are constantly changing. The present study used GEO and TCGA databases to analyze mRNA alterations, and search for efficient diagnostic and prognostic biomarkers in ccRCC.

In the present study, four ccRCC datasets (GSE46699, GSE36895, GSE53000 and GSE53757) were downloaded from the GEO. Subsequently, the differentially expressed genes (DEGs) were screened by comparing adjacent normal kidney samples with ccRCC samples. The Robust Rank Aggregation (RRA) method was used to identify the statistically significant genes based on their ranks in each profile. Tumor-associated genetic alterations are known to result in tumorigenesis and the progression of ccRCC (12). Therefore, the present study used the tumor-associated gene (TAG) database to identify the DEGs associated with cancer. Through this analysis, Erb-B2 receptor tyrosine kinase 4 (ERBB4), centrosomal protein 55 (CEP55) and vascular endothelial growth factor A (VEGFA) were identified as oncogenes. Furthermore, all three oncogenes were associated with the stage of ccRCC, whereas only CEP55 was associated with tumor prognosis according to Gene Expression Profiling Interactive Analysis (GEPIA). The oncogenic role of CEP55 in ccRCC remains unclear. TCGA data were used to analyze the expression levels, and potential diagnostic and prognostic values of CEP55 in ccRCC. Gene set enrichment analysis (GSEA) was also used to explore the underlying molecular mechanisms of CEP55 in ccRCC. The present study revealed that CEP55 may serve as a novel potential diagnostic and prognostic marker, and a potential therapeutic target for ccRCC.

\section{Materials and methods}

Microarray and TCGA ccRCC data. The key phrase 'renal cell carcinoma' was used to search GEO datasets (www.ncbi. nlm.nih.gov/geo), and the GSE46699, GSE36895, GSE53000 and GSE53757 microarray datasets were downloaded. The GSE46699 data were obtained using the GPL570 platform and included 63 adjacent normal kidney specimens and $67 \mathrm{ccRCC}$ samples. The platform used to obtain the GSE36895 data was also GPL570, and this dataset consisted of 23 adjacent normal kidney samples and 29 ccRCC samples. The GSE53000 data were obtained using the GPL6244 platform and included six adjacent normal kidney samples and 28 ccRCC samples. The platform used to obtain the GSE53757 data was GPL570, and this dataset consisted of 72 adjacent normal kidney samples and $72 \mathrm{ccRCC}$ samples. The data were calibrated, standardized and $\log _{2}$ transformed. TCGA contains DNA, RNA and protein data for human cancers, and can be used to analyze expression of these components in various types of cancer (10).
In the present study, the RNA-sequencing (RNA-seq) data of patients diagnosed with ccRCC were downloaded from TCGA on May 9th, 2018. The RNA-seq data based on the Illumina HiSeq RNA-seq platform included 74 adjacent normal kidney tissues and $536 \mathrm{ccRCC}$ tissues. Additionally, there were $416 \mathrm{ccRCC}$ patients with full clinical information including age, sex, histological type, Tumor-Node-Metastasis stage (13) and overall survival (OS) time. The dataset information is presented in Table I (14-17). R software (version 3.5.0, https://www.r-project.org/) was used for further analysis.

Integration of microarray data. Limma package analysis (18) was used to identify DEGs from the four microarray datasets. The RRA package (19) from R language was used to analyze the list of up- and downregulated genes from the four profiles. The RRA method is used to compare the expression of different genes based on their ranks (20). The higher the rank of a gene is in the selected datasets, the smaller its P-value is (21).

Functional annotation of DEGs. The present study downloaded all of the tumor-associated genes from the TAG database to assess whether the screened DEGs were associated with cancer (22). Subsequently, GEPIA (http://gepia. cancer-pku.cn/) was applied to validate the different expression and prognostic values of the selected genes in ccRCC.

Reverse transcription-polymerase chain reaction (RT-PCR). A total of 15 paired fresh ccRCC tissues and adjacent tissues were obtained from patients (nine men and six women; age, $58 \pm 11$ years). All patients underwent partial or radical nephrectomy at the Department of Urology, Ningbo Medical Centre Lihuili Hospital (Ningbo, China) between May and June 2018. Written informed consent was obtained from the patients and the present study was approved by the Ethics Committee of Ningbo Medical Centre Lihuili Hospital. RNA extraction, reverse transcription and amplification were conducted according to our previous study (5). The primers used were as follows: CEP55, forward 5'-GCCATTGGGCGA GACCTACCT-3', reverse 5'-GTTCGGGACTTCGCTCAC CTT-3'; and GAPDH, forward 5'-ACAACTTTGGTATCG TGGAAGG-3' and reverse 5'-GCCATCACGCCACAGTTT C-3'. GAPDH was used as the control. Data were presented as the means \pm standard deviation from three experiments and were analyzed using the $2^{-\Delta \Delta \mathrm{Cq}}$ method (23).

Diagnosis analysis. All 610 adjacent normal kidney and ccRCC tissue samples from TCGA dataset were randomly and equally divided into the training set (total $n=305$; number of adjacent normal kidney samples $=37$; and number of ccRCC samples $=268$ ) and the validation set (total $n=305$; number of adjacent normal kidney samples $=37$; and number of ccRCC samples $=268$ ). The training set was used to identify the potential diagnostic value of CEP55 expression, and the validation set was used to verify it. Area under the curve (AUC) values obtained from the receiver operating characteristic (ROC) curve analysis were utilized to assess the diagnostic effectiveness of CEP55. Generally, an AUC value of $>0.85$ is considered to indicate diagnostic value (24). The cut-off value is the detection value corresponding to the maximum value of the sum of sensitivity and specificity. 
Table I. Summary of GEO and TCGA clear cell renal cell carcinoma datasets.

\begin{tabular}{|c|c|c|c|c|c|c|}
\hline Author, year & Sample & GEO no. & Platform & Normal & Tumor & (Refs.) \\
\hline Eckel-Passow et al, 2014 & ccRCC & GSE46699 & GPL570 & 63 & 67 & (14) \\
\hline Peña-Llopis et al, 2012 & ccRCC & GSE36895 & GPL570 & 23 & 29 & $(15)$ \\
\hline Gerlinger et al, 2014 & $\mathrm{ccRCC}$ & GSE53000 & GPL6244 & 6 & 28 & (16) \\
\hline von Roemeling et al, 2014 & $\mathrm{ccRCC}$ & GSE53757 & GPL570 & 72 & 72 & (17) \\
\hline TCGA & $\operatorname{ccRCC}$ & - & - & 74 & 536 & - \\
\hline
\end{tabular}

GEO, Gene Expression Omnibus; TCGA, The Cancer Genome Atlas.

GSEA. The c2.cp.kegg.gmt annotated gene set from the Java GSEA application was used to conduct the Kyoto Encyclopedia of Genes and Genomes (KEGG) enrichment analysis $(25,26)$. Normal $\mathrm{P}<0.05$ and false discovery rate q-value $<0.25$ were chosen to indicate statistical significance.

Statistical analysis. Student's t-test was used to analyze the significance between two groups. The associations between CEP55 and clinical factors were analyzed by $\chi^{2}$ test. Spearman's rank correlation coefficient was performed to access bivariate correlations. In addition, Kaplan-Meier analysis was used to assess OS and significance was determined using the log-rank test. Furthermore, the prognostic value of CEP55 in patients with ccRCC was evaluated by univariate and multivariate Cox regression analyses (27). All tests were two-sided and $\mathrm{P}<0.05$ was considered to indicate a statistically significant difference. All statistical analyses were carried out using SPSS 23.0 (IBM, Corp., Armonk, NY, USA) and GraphPad Prism 7.0 (GraphPad Software, Inc., La Jolla, CA, USA).

\section{Results}

Microarray data resources and DEGs in ccRCC. The ccRCC expression microarray datasets GSE46699, GSE36895, GSE53000 and GSE53757 were downloaded from the GEO and were analyzed using limma package (adjusted $\mathrm{P}<0.05$, $\mid \log \mathrm{FCl}>1)$. A total of $271 \mathrm{DEGs}$ were screened from the GSE46699; of which, 104 and 167 genes were up- and downregulated, respectively. In addition, 100 upregulated genes and 290 downregulated genes were obtained from the GSE36895 dataset. Furthermore, 532 upregulated genes and 649 downregulated genes, respectively, were detected from the GSE53000 dataset. Overall, there were 1,552 upregulated genes and 1,821 downregulated genes among the 3,373 DEGs screened from the GSE53757 dataset. All of the DEGs from the four microarrays are presented in Fig. 1.

Identification of DEGs in ccRCC by integrated bioinformatics. DEGs from each of the four ccRCC gene microarrays were identified based on the $\log \mathrm{FC}$ value and then analyzed using the RRA method. A total of 223 DEGs were identified, including 78 upregulated genes and 145 downregulated genes according to the cut-off criterion of corrected $\mathrm{P}<0.05$ (Table II). The heatmap presents the top 55 upregulated and 20 downregulated genes (Fig. 2).
Functional annotation of DEGs. To determine whether the DEGs screened by RRA were associated with cancer, the present study searched the TAG database. The results revealed that 12 genes were TAGs; of which, three were oncogenes, including ERBB4, CEP55 and VEGFA; six were tumor suppressor genes, including ETS homologous factor (EHF), caveolin 1 (CAV1), S100 calcium binding protein A2 (S100A2), lysyl oxidase (LOX), caveolin 2 (CAV2) and transforming growth factor $\beta$ induced (TGFBI); and three were uncertain, including solute carrier family 6 member 3 (SLC6A3), angiopoietin-like 4 (ANGPTL4) and fibroblast growth factor 9 (FGF9). Furthermore, all of the three oncogenes were associated with ccRCC stage (Fig. 3), whereas, of the three oncogenes, only CEP55 was associated with OS and disease-free survival according to GEPIA (Fig. 4).

High expression of CEP55 in ccRCC. The molecular mechanisms, and the diagnostic and prognostic value of CEP55 in ccRCC, remain unclear. Therefore, the present study focused on the CEP55 gene for further study. As shown in Fig. 5A, CEP55 was upregulated in ccRCC tissues $(n=536)$ but downregulated in adjacent normal kidney tissues $(\mathrm{n}=74)(\mathrm{P}<0.0001)$ based on the publicly available TCGA data. The present study further validated the overexpression of CEP55 in ccRCC tissues based on 71 paired ccRCC tissues $(\mathrm{P}<0.001)$ (Fig. 5B). Furthermore, the mRNA expression levels of CEP55 were significantly upregulated in 15 paired clinical ccRCC tissues, as determined by RT-PCR analysis ( $\mathrm{P}<0.05$; Fig. $5 \mathrm{C})$. These results indicated that CEP55 was upregulated and may serve essential roles in the occurrence of ccRCC.

Diagnostic value of CEP55 in ccRCC. To validate the potential diagnostic value of CEP55 mRNA, the training set was initially studied, and then the validation set was utilized for verification. ROC curves revealed that the AUC was 0.897 [confidence interval (CI): 0.817-0.975] in the training set and 0.947 (CI: 0.891-1.003) in the validation set for CEP55 (Fig. 6). Applying the cut-off value of 0.437 , the specificity and sensitivity of CEP55 for the diagnosis of ccRCC patients vs. healthy controls was $92.7 \%$ (95\% CI: 90.2-94.8\%) and 86.5\% (95\% CI: 76.6-93.3\%), respectively. These results confirmed that CEP55 could act as a diagnostic biomarker for ccRCC tissues.

Association between CEP55 and clinical factors in ccRCC. To verify the association between CEP55 and clinical factors 
Table II. DEGs in clear cell renal cell carcinoma, as determined using the Robust Rank Aggregation method.

DEGs

Upregulated

Downregulated

A

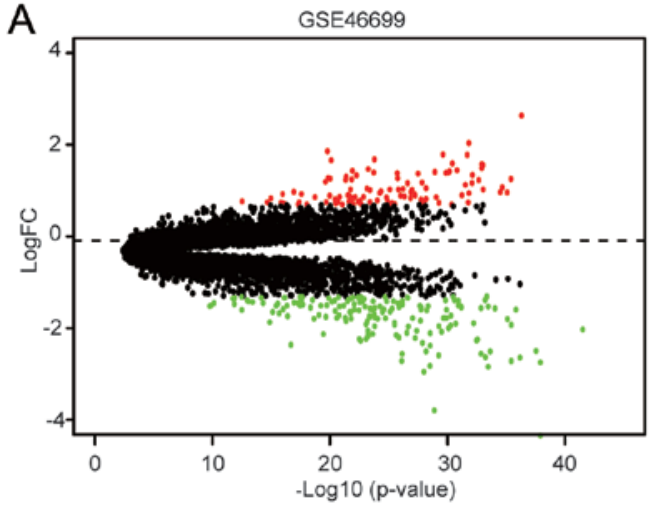

C

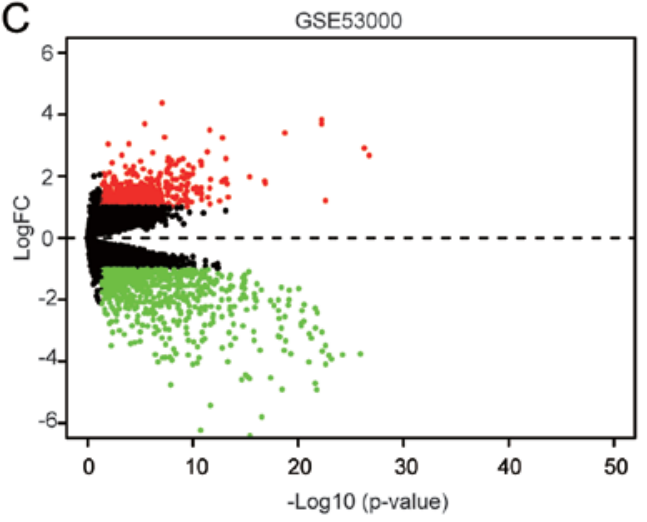

Gene names

DIRAS2, MCAM, IGFBP3, FABP5, C1QA, LAMA4, TNFAIP6, NDUFA4L2, VCAN, CXCR4, CEP55, CD163, NPTX2, IDO1, ITGB2, GAL3ST1, CTHRC1, C1QC, HK2, VEGFA, SEMA5B, APOLD1, DNAH11, SCARB1, RASSF2, LOX, AHNAK2, COL23A1, GAS2L3, NETO2, FABP7, CCL18, CAV1, LINC00462, CXCL13, TGFBI, PLK2, ANGPTL4, DDIT4, INHBB, QRFPR, LAPTM5, BHLHE41, CYP2J2, SPAG4, C5orf46, LPCAT1, PNMA2, CP, ADM, HILPDA, LINC00887, LOXL2, PLA2G7, NNMT, CA9, C1QB, APOC1, ADAMDEC1, ENPP3, EGLN3, ENO2, TLR3, FCGR1B, TMEM45A, PCSK6, CD70, KISS1R, CENPK, CAV2, SLC6A3, RGS1, VWF, FN1, ANGPT2, ST8SIA4, C3, ESM1

CYP17A1, SMIM24, NPHS2, ERBB4, ESRP1, PSAT1, RALYL, SLC4A9, TFCP2L1, XPNPEP2, SPINK1, SCNN1B, ALB, PLG, SLC4A1, SLC12A1, ALDOB, CNTN3, ERP27, ALDH8A1, TSPAN8, UPP2, FCAMR, LOC284578, SLC22A8, KL, KCNJ10, EHF, SLC13A3, CYP8B1, AZGP1, ALDH6A1, HPD, SLC7A13, SLC22A6, EFHD1, SUCNR1, TMEM213, AFM, ACPP, LOC100505985, RAB25, HPGD, CRHBP, FXYD4, HRG, ASS1, AQP2, DAO, SLC16A9, DNER, C16orf89, DCXR, EGF, PVALB, RHCG, UMOD, FOXI1, CYP4A11, FAM3B, ABAT, HOGA1, ATP6V0D2, LINC00645, TMEM52B, NRK, PTH1R, TMEM207, TMEM174, GGT6, CALB1, DIO1, KCNJ1, MIOX, TUBB2B, SLC12A3, ATP6V1G3, CRYAA, RNF212B, SCNN1A, HSD11B2, TMEM178A, OGDHL, TUBAL3, SOSTDC1, TYRP1, S100A2, ENPP6, MAL, PCP4, DEFB1, FGF1, ATP6V1B1, SERPINA5, DUSP9, SFRP1, VTCN1, TFAP2B, GLDC, GPC5, FBP1, CLDN8, CLCNKB, SLC7A8, PPP1R1A, SLC22A12, PIPOX, CLDN16, PLPPR1, MAL2, CYP2B6, NPHS1, CYP27B1, DDC, TMPRSS2, PRODH2, FABP1, CYP4F3, CDH16, PCK1, MTTP, SLC5A2, SLC22A7, ESRRG, SLC26A4, SLC47A2, FAM151A, ADH1C, EPCAM, KNG1, CYP4F2, ADH6, PCSK1N, MT1H, ATP6V0A4, SLC34A1, MUC15, HEPACAM2, RP11-999E24.3, FGF9, DPEP1, AGPAT9, SOST, LRRC19, ACSF2

DEGs, differentially expressed genes.

B

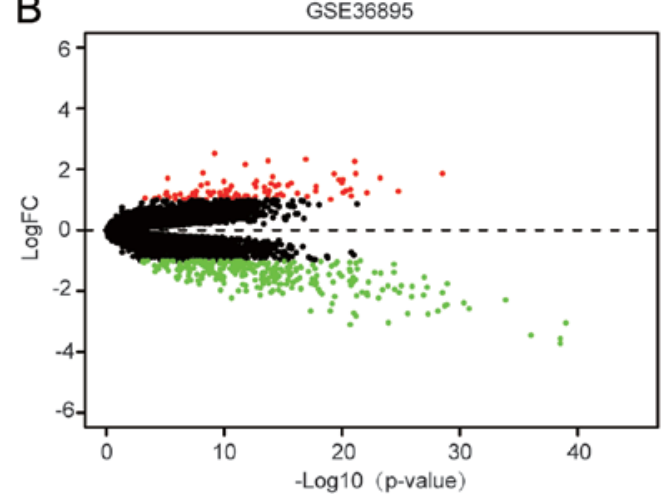

D

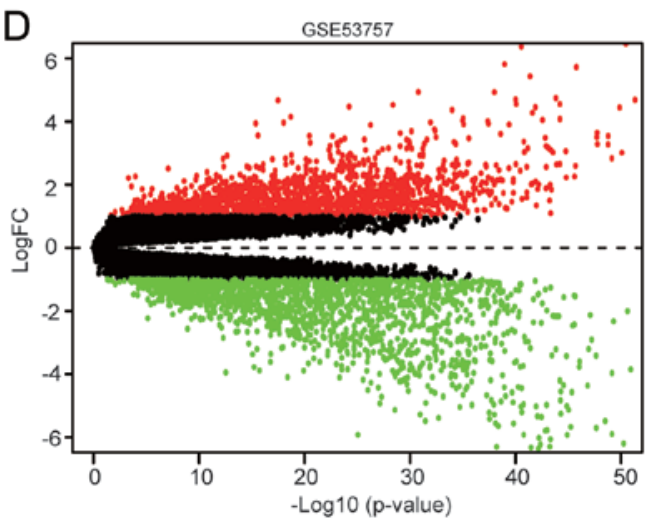

Figure 1. Volcano plots of the aberrantly expressed genes between ccRCC tissues and adjacent normal kidney tissues in the four datasets. (A) GSE46699, (B) GSE36895, (C) GSE53000 and (D) GSE53757 data. Red dots represent upregulated genes defined as logFC $>1.0$ and adjusted P<0.05. Green dots indicate downregulated genes based on $\log \mathrm{FC}<-1.0$ and adjusted $\mathrm{P}<0.05$. Black dots represent $\mathrm{mRNA}$ expression with $\mid \log \mathrm{FCl}<1$ and adjusted $\mathrm{P}>0.05$. ccRCC, clear cell renal cell carcinoma; FC, fold change. 


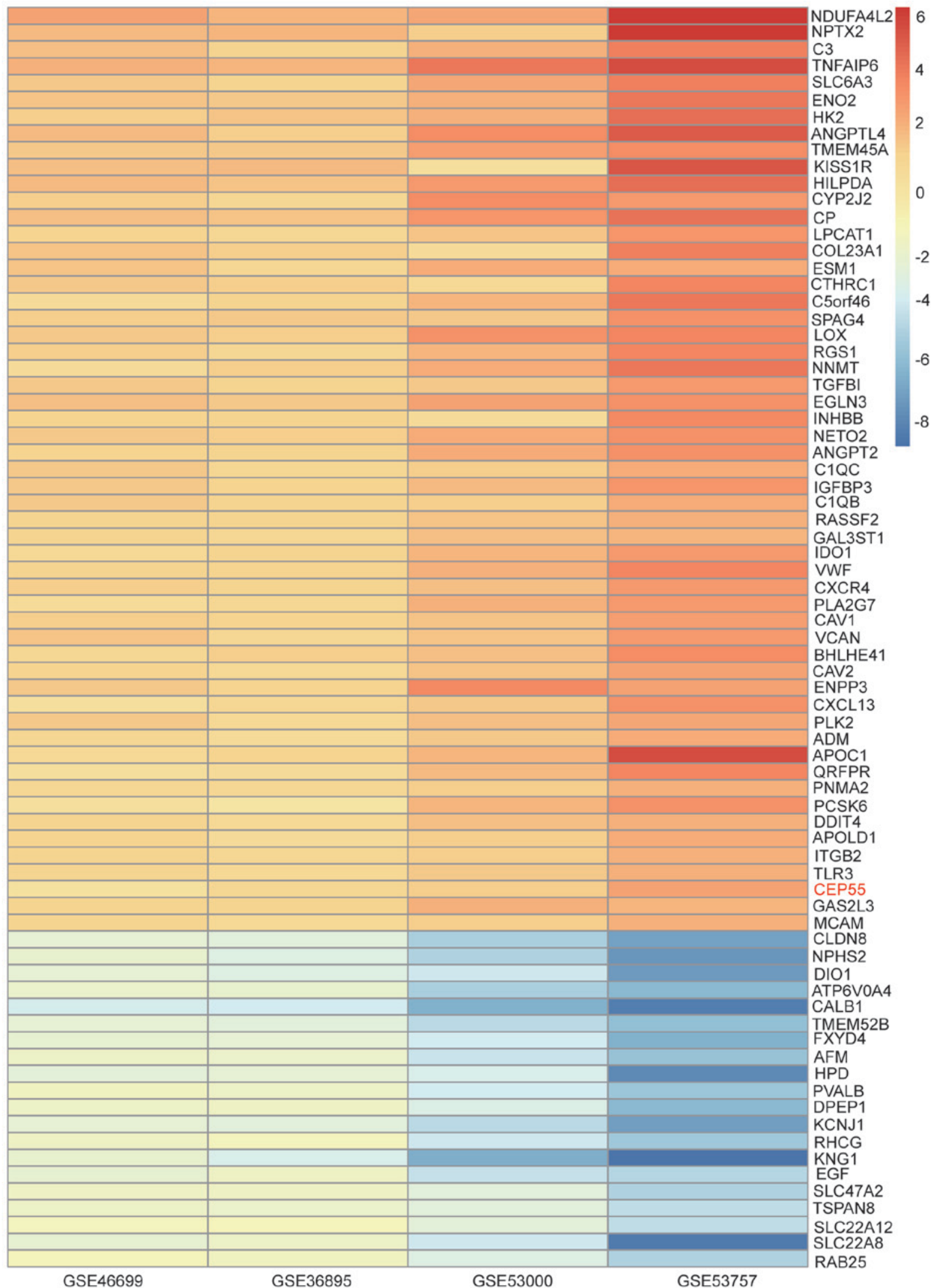

Figure 2. LogFC heatmap of each expression microarray. The color label indicates the different logFC values. Abscissa, Gene Expression Omnibus accession number; ordinate, gene names. FC, fold change.

in ccRCC, the 416 ccRCC patients with full clinical information in TCGA dataset were further analyzed. The mRNA expression levels of CEP55 were strongly associated with sex $(\mathrm{P}=0.001)$, histological grade $(\mathrm{P}<0.001)$, stage $(\mathrm{P}<0.001)$, T classification $(\mathrm{P}<0.001), \mathrm{N}$ classification $(\mathrm{P}=0.007), \mathrm{M}$ classification $(\mathrm{P}=0.002)$ and vital status $(\mathrm{P}<0.001)$, but not with 

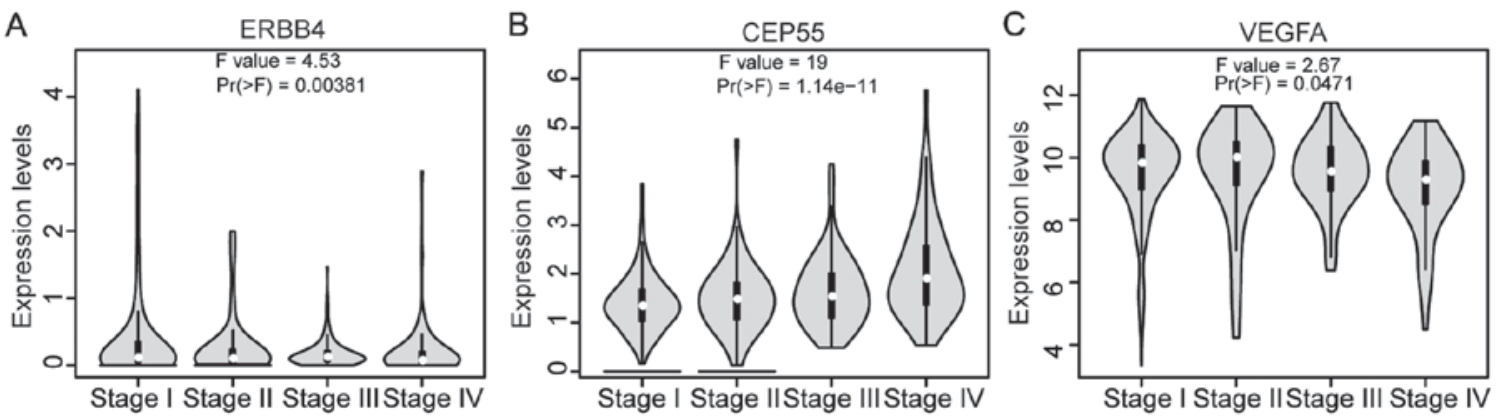

Figure 3. Associations between the expression of (A) ERBB4, (B) CEP55 and (C) VEGFA, and the stage of clear cell renal cell carcinoma, as determined by Gene Expression Profiling Interactive Analysis. CEP55, centrosomal protein 55; ERBB4, Erb-B2 receptor tyrosine kinase 4; VEGFA, vascular endothelial growth factor A.
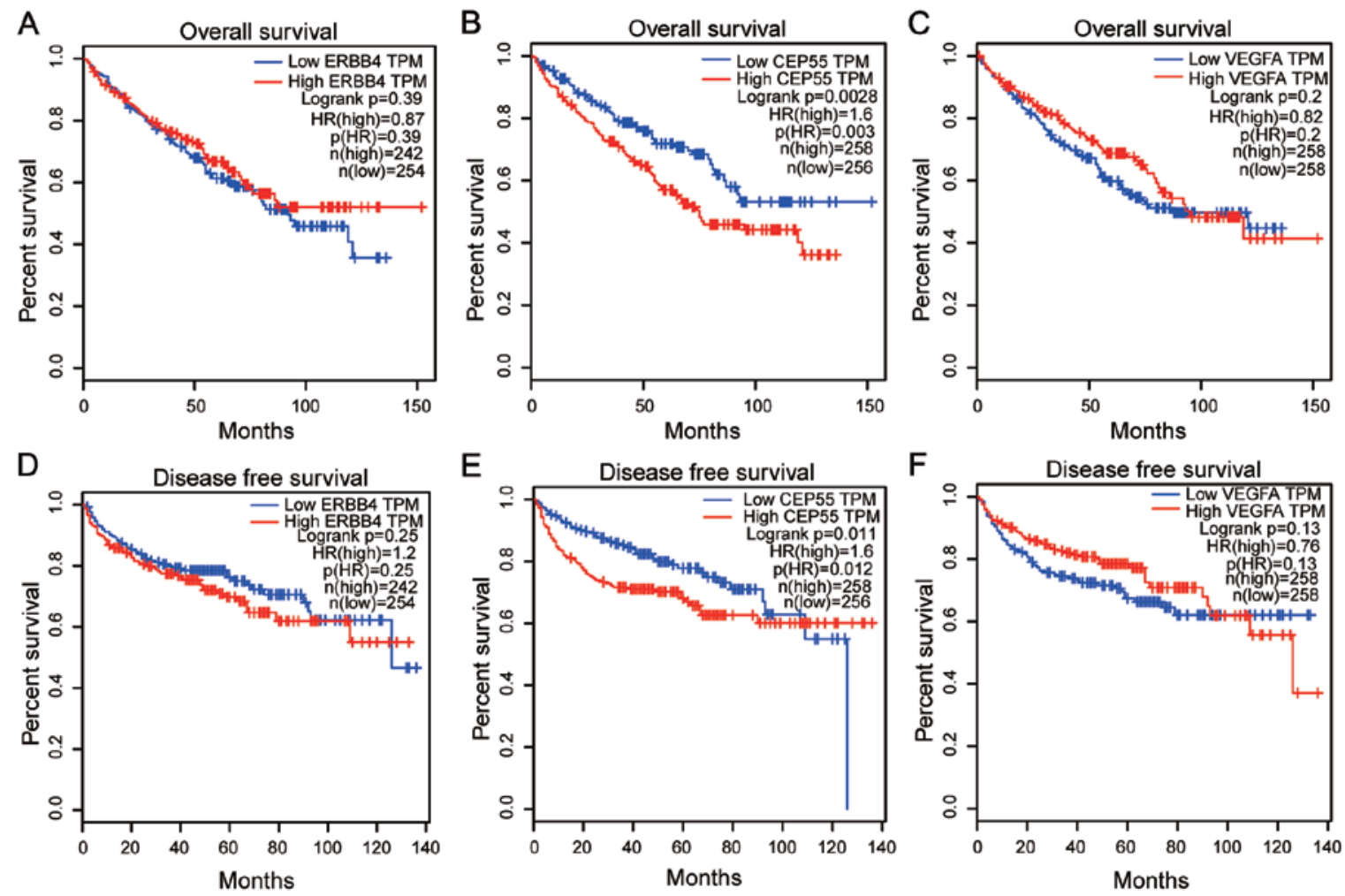

Figure 4. Kaplan-Meier survival curve analysis. Association between three oncogenes and overall and disease-free survival of clear cell renal cell carcinoma was determined by Gene Expression Profiling Interactive Analysis. Overall survival analysis: (A) ERBB4, (B) CEP55 and (C) VEGFA. Disease-free survival analysis: (D) ERBB4, (E) CEP44 and (F) VEGFA. CEP55, centrosomal protein 55; ERBB4, Erb-B2 receptor tyrosine kinase 4; HR, hazard ratio; VEGFA, vascular endothelial growth factor A.

age $(\mathrm{P}=0.922$; Table III). As determined by Spearman's analysis, CEP55 was correlated with sex $(\mathrm{P}=0.002)$, histological grade $(\mathrm{P}<0.001)$, stage $(\mathrm{P}<0.001)$, $\mathrm{T}$ classification $(\mathrm{P}<0.001)$, $\mathrm{N}$ classification $(\mathrm{P}<0.001)$, M classification $(\mathrm{P}<0.001)$ and vital status $(\mathrm{P}<0.001$; Table IV). These results revealed a potential association between high CEP55 expression levels and the aggressive clinical characteristics of ccRCC, and also with sex.

Association between high expression of CEP55 and poor prognosis. In order to further investigate the upregulation of CEP55 as a prognostic factor, 416 patients with ccRCC from TCGA were analyzed. Cox regression analysis was performed to determine whether CEP55 could be a potential predictive and prognostic factor. As shown in Table V, high CEP55 expression was associated with a significantly increased risk of mortality in patients with ccRCC $(\mathrm{P}=0.006)$ when compared to those with low CEP55 expression, as determined by univariate Cox regression analysis. Multivariate Cox regression analysis also revealed that $\mathrm{CEP} 55$ expression $(\mathrm{P}=0.011)$ and $\mathrm{M}$ classification $(\mathrm{P}<0.001)$ could be factors for predicting poor prognosis in ccRCC (Table V). These results indicated that CEP55 expression may serve as a prognostic biomarker in ccRCC.

GSEA. To evaluate KEGG pathway enrichment of CEP55 expression, GSEA was conducted based on the median expression of CEP55 in GSE53757. The top 10 relevant pathways in the CEP55 high-expression group are shown in Table VI. Taken together, the present GSEA results suggested that 
A

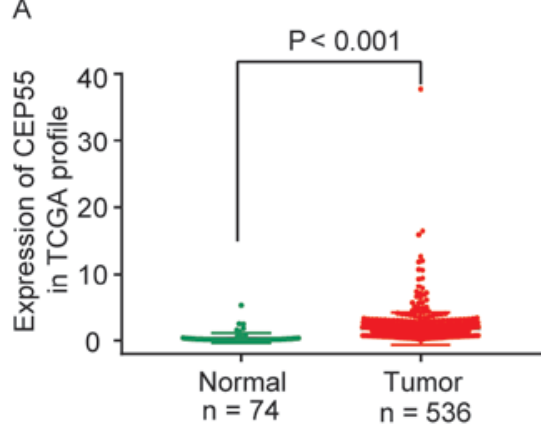

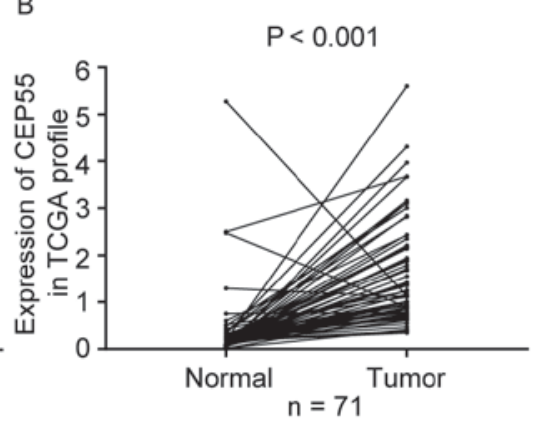

C

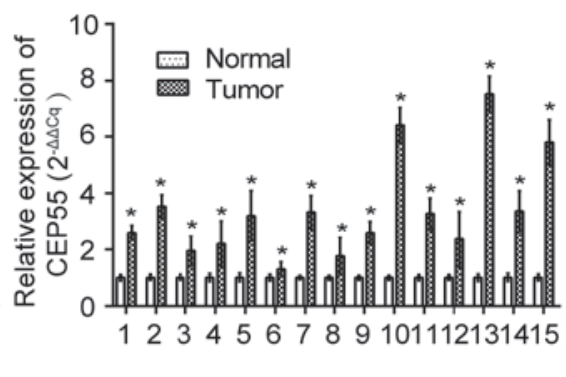

Figure 5. Overexpression of CEP55 in ccRCC. (A) CEP55 mRNA was upregulated in 536 ccRCC tissues when compared with 74 adjacent normal kidney tissues according to TCGA data. (B) CEP55 mRNA expression was significantly increased in 71 paired ccRCC tissues compared with adjacent normal tissues, as determined using TCGA data. (C) Reverse transcription-polymerase chain reaction analysis of CEP55 expression in 15 paired ccRCC tissues and their adjacent normal tissues. Data are representative of three experiments. ${ }^{*} \mathrm{P}<0.05$. CEP55, centrosomal protein 55; ccRCC, clear cell renal cell carcinoma; TCGA, The Cancer Genome Atlas.
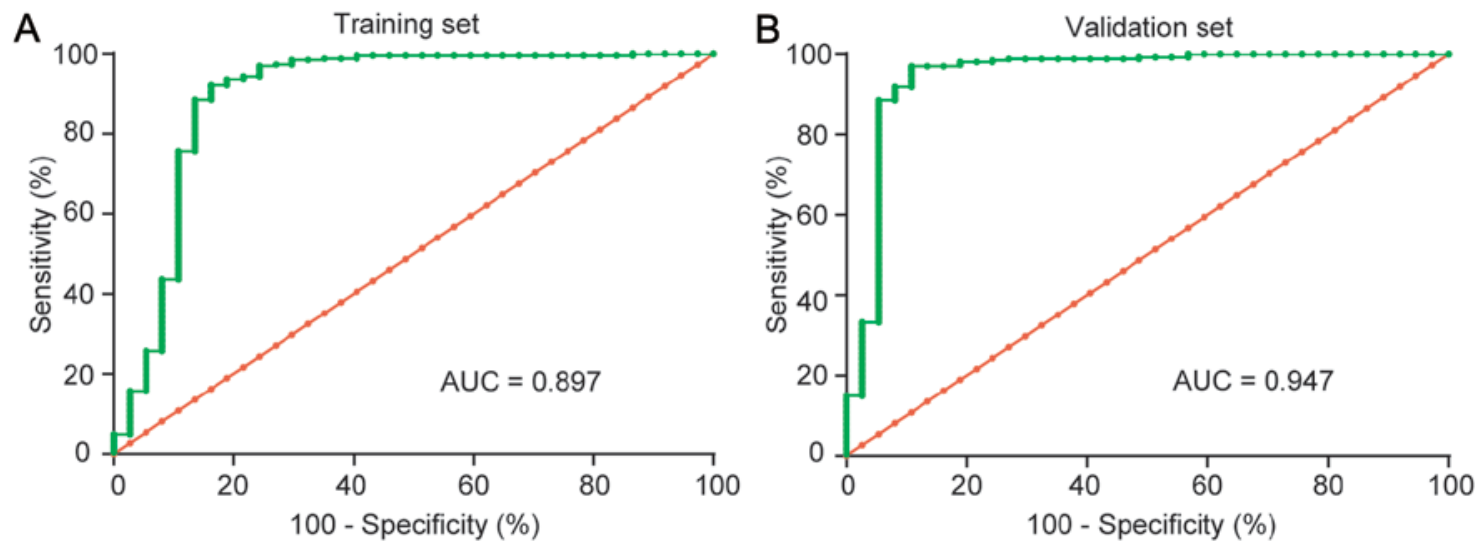

Figure 6. Receiver operating characteristic curves of centrosomal protein 55 in the (A) training and (B) validation sets. AUC, area under the curve.

CEP55 may have multifaceted functions in immunization, cell adhesion, inflammation, the Janus kinase/signal transducer and activator of transcription (JAK/STAT) signaling pathway and cell proliferation in kidney carcinogenesis and progression (Fig. 7).

\section{Discussion}

ccRCC is the most common subtype of RCC, which is associated with an increasing incidence, high metastatic potential and high mortality rates (28). Over recent decades, the 5-year survival rate of ccRCC remains poor, despite in-depth study of the underlying mechanisms of occurrence, progression and metastasis $(29,30)$. Although numerous targeted and immunosuppressive drugs have emerged, drug resistance is still an urgent problem to be solved in the treatment of ccRCC. Therefore, highly specific and sensitive biomarkers, and elucidation of the underlying mechanism, are urgently required to reduce the mortality risk for patients with ccRCC.

Bioinformatics is a multidisciplinary field of research, and bioinformatics analysis is specifically utilized to identify candidate genes that aid the understanding of the genetic basis of diseases. In the present study, four gene expression profiles from the GEO were integrated and further analyzed via bioinformatics analysis. A total of 223 DEGs were screened, including 78 upregulated and 145 downregulated genes, by the RRA method. In addition, 12 genes that were associated with cancer were identified from DEGs according to the TAG database, including ERBB4, CEP55, VEGFA, EHF, CAV1, S100A2, LOX, CAV2, TGFBI, SLC6A3, ANGPTL4 and FGF9. Furthermore, only CEP55 was closely associated with ccRCC progression and prognosis as determined by GEPIA, an online method based on TCGA. Therefore, CEP55 has the potential to be a novel and valuable oncotarget protein in ccRCC.

CEP55, also known as FLJ10540, C10orf3 or URCC6, is located on chromosome 10q23.33 and serves an important role in cytokinesis $(31,32)$. As described previously, the protein localizes to the centrosome during interphase, dissociates from the centrosome in the $M$ phase, and condenses to the midbody during cytokinesis (33). However, centrosome amplification may participate in the origin of chromosomal instability during tumor development. High CEP55 expression results in disordered cytokinesis and an increased number of unstable binucleated cells; these are oncogenic characteristics in tumorigenesis $(34,35)$. Numerous studies have demonstrated that overexpression of CEP55 is associated with the pathological processes of several human malignances, including hepatocellular carcinoma (36-38), colon carcinoma (39), breast cancer (40-42), lung cancer $(43,44)$, glioma $(45-47)$, nasopharyngeal carcinoma (48), oral cavity squamous cell carcinoma (49), 
Table III. Association between CEP55 expression and clinical characteristics of patients with clear cell renal cell carcinoma in The Cancer Genome Atlas dataset.

\begin{tabular}{|c|c|c|c|c|}
\hline \multirow[b]{2}{*}{ Characteristics } & \multirow[b]{2}{*}{ Number of cases $(\%)$} & \multicolumn{2}{|c|}{ CEP55 } & \multirow[b]{2}{*}{ P-value } \\
\hline & & Low & High & \\
\hline Age (years) & & & & 0.922 \\
\hline$\geq 60$ & $215(51.7)$ & 107 & 108 & \\
\hline$<60$ & $201(48.3)$ & 101 & 100 & \\
\hline Sex & & & & 0.001 \\
\hline Male & $264(63.5)$ & 116 & 148 & \\
\hline Female & $152(36.5)$ & 92 & 60 & \\
\hline \multicolumn{5}{|c|}{ Histological grade } \\
\hline $\mathrm{G} 1$ & $10(2.4)$ & 9 & 1 & $<0.001$ \\
\hline $\mathrm{G} 2$ & $201(48.3)$ & 116 & 85 & \\
\hline G3 & $160(38.5)$ & 70 & 90 & \\
\hline G4 & $45(10.8)$ & 13 & 32 & \\
\hline Stage & & & & $<0.001$ \\
\hline I & $252(60.6)$ & 151 & 101 & \\
\hline II & $53(12.7)$ & 25 & 28 & \\
\hline III & $69(16.6)$ & 21 & 48 & \\
\hline VI & $42(10.1)$ & 11 & 31 & \\
\hline T classification & & & & $<0.001$ \\
\hline $\mathrm{T} 1$ & $256(61.5)$ & 152 & 104 & \\
\hline $\mathrm{T} 2$ & $61(14.7)$ & 28 & 33 & \\
\hline $\mathrm{T} 3$ & $92(22.1)$ & 28 & 64 & \\
\hline $\mathrm{T} 4$ & $7(1.7)$ & 0 & 7 & \\
\hline $\mathrm{N}$ classification & & & & 0.007 \\
\hline N0 & 402 (96.6) & 206 & 196 & \\
\hline N1-2 & $14(3.4)$ & 2 & 12 & \\
\hline M classification & & & & 0.002 \\
\hline M0 & $375(90.1)$ & 197 & 178 & \\
\hline M1 & $41(9.9)$ & 11 & 30 & \\
\hline \multicolumn{5}{|l|}{ Vital status } \\
\hline Alive & $308(74)$ & 170 & 138 & $<0.001$ \\
\hline Deceased & $108(26)$ & 38 & 70 & \\
\hline
\end{tabular}

Data were analyzed using the $\chi^{2}$ test. CEP55, centrosomal protein 55; M, metastasis; N, node; T, tumor.

osteosarcoma (50), head and neck cancer (51), anaplastic thyroid carcinoma (52), cervical cancer (53), esophageal squamous cell carcinoma $(54,55)$, pancreatic cancer $(56)$ and prostate cancer $(57,58)$. In addition, high CEP55 expression in colorectal cancer downregulates the tumor susceptibility 101 gene in a post-transcriptional manner, and knockdown of CEP55 inhibits cell growth and increases apoptosis (39). CEP55 is significantly upregulated in metastatic and recurrent prostate cancer when compared with localized and non-recurrent prostate cancer, and is associated with a poor biochemical recurrence-free survival time following radical prostatectomy (58). High CEP55 and osteopontin expression levels in nasopharyngeal carcinoma tissues are markedly associated with advanced tumor stages and poor 5-year survival time, and result in the acceleration of cell growth and motility (48). In addition, patients with hepatocellular carcinoma (HCC) and higher CEP55 expression have poorer
Table IV. Correlation of CEP55 mRNA expression with clinical characteristics, as determined by Spearman analysis.

\begin{tabular}{lcr}
\hline & \multicolumn{2}{c}{ CEP55 expression level } \\
\cline { 2 - 3 } Characteristics & Spearman correlation & P-value \\
\hline Age & -0.077 & 0.119 \\
Sex & 0.152 & 0.002 \\
Histological grade & 0.271 & $<0.001$ \\
Stage & 0.326 & $<0.001$ \\
T classification & 0.311 & $<0.001$ \\
N classification & 0.224 & $<0.001$ \\
M classification & 0.228 & $<0.001$ \\
Vital status & 0.217 & $<0.001$ \\
\hline
\end{tabular}

CEP55, centrosomal protein 55; M, metastasis; $\mathrm{N}$, node; $\mathrm{T}$, tumor. 
Table V. Univariate and multivariate Cox regression analyses of various prognostic factors in patients with clear cell renal cell carcinoma.

\begin{tabular}{lccccccc}
\hline & \multicolumn{3}{c}{ Univariate analysis } & & \multicolumn{3}{c}{ Multivariate analysis } \\
\cline { 2 - 4 } Characteristics & P-value & HR & $95 \%$ CI & & P-value & HR & $95 \%$ CI \\
\hline CEP55 expression & 0.006 & 1.755 & $1.179-2.612$ & & 0.011 & 1.683 & $1.127-2.513$ \\
Age & 0.002 & 1.919 & $1.276-2.884$ & & - & - & - \\
Sex & 0.450 & 0.861 & $0.583-1.270$ & & - & - & - \\
Histological grade & $<0.001$ & 2.429 & $1.847-3.195$ & & - & - & - \\
Stage & $<0.001$ & 2.000 & $1.700-2.353$ & & - & - & - \\
T classification & $<0.001$ & 2.133 & $1.744-2.610$ & & - & - & - \\
N classification & $<0.001$ & 4.184 & $2.104-8.320$ & & - & - & $3.823-9.043$ \\
M classification & $<0.001$ & 6.005 & $3.912-9.216$ & & $<0.001$ & 5.880 & \\
\hline
\end{tabular}

CEP55, centrosomal protein 55; CI, confidence interval; HR, hazard ratio; M, metastasis; N, node; T, tumor.

Table VI. KEGG pathways associated with high CEP55 expression, as determined by gene set enrichment analysis.

\begin{tabular}{lccccc}
\hline KEGG pathway & Size & ES & NES & NOM P-value & FDR q-value \\
\hline Natural killer cell-mediated cytotoxicity & 127 & 0.66 & 2.28 & $<0.001$ & $<0.001$ \\
Chemokine signaling pathway & 180 & 0.59 & 2.17 & $<0.001$ & 0.002 \\
Cell adhesion molecules CAMs & 128 & 0.56 & 2.11 & $<0.001$ & 0.005 \\
Cytokine-cytokine receptor interaction & 246 & 0.56 & 2.1 & $<0.001$ & 0.003 \\
Antigen processing and presentation & 73 & 0.64 & 2.06 & $<0.001$ & 0.004 \\
Toll-like receptor signaling pathway & 96 & 0.6 & 2.03 & $<0.001$ & 0.004 \\
T cell receptor signaling pathway & 107 & 0.57 & 2.02 & 0.004 & 0.004 \\
B cell receptor signaling pathway & 74 & 0.57 & 1.97 & 0.004 & 0.006 \\
JAK/STAT signaling pathway & 149 & 0.51 & 1.95 & $<0.001$ & 0.006 \\
Cell cycle & 122 & 0.54 & 1.91 & $<0.001$ & 0.010 \\
\hline
\end{tabular}

CEP55, centrosomal protein 55; ES, enrichment score; FDR, false discovery rate; GSEA, gene set enrichment analysis; KEGG, Kyoto Encyclopedia of Genes and Genomes; NES, normal enrichment score; NOM, normal.

survival (37). CEP55 is associated with several oncogenic functions, including anchorage-independent growth, increased cell growth during starvation and induction of tumorigenesis in nude mice (36). A previous mechanistic study of CEP55-mediated oncogenesis indicated that it functions though interactions with phosphoinositide-3 kinase (7). Jones et al (59) revealed that CEP55 is one of 18 genes that is positively correlated with renal cancer progression and is highly expressed in patients with RCC and metastasis. In addition, Luo et al (60) analyzed three datasets, including GSE36895 and GSE46699; however, CEP55 was not revealed to be a DEG, this may be due to the different methods of analysis used compared with those used in the present study. The increased expression and carcinogenic properties of CEP55, and its association with clinical outcome in patients with ccRCC, remains unclear. Therefore, the present study used GEO and TCGA datasets to determine the expression and clinical significance of CEP55 in ccRCC.
In the present study, the results revealed that CEP55 was highly expressed in ccRCC tissues based on the GEO and TCGA datasets. In addition, the RT-PCR analysis demonstrated that the mRNA expression levels of CEP55 in 15 paired clinical ccRCC samples were consistent with the results obtained from open databases. This indicated that using open databases could support novel research design. The ROC curve analysis indicated that CEP55 may be a diagnostic biomarker for ccRCC with an AUC of 0.897 in the training set and 0.947 in the validation set. The specificity and sensitivity of CEP55 for the diagnosis of patients with ccRCC was 92.7 and $86.5 \%$, respectively. In addition, CEP55 mRNA expression was markedly associated with clinical characteristics, including sex, histological grade, stage, T classification, $\mathrm{N}$ classification, $\mathrm{M}$ classification and OS time. The univariate and multivariate analyses also demonstrated that CEP55 may be an independent prognostic factor in patients with ccRCC. From these results, it was suggested that CEP55 may be considered a novel 
A

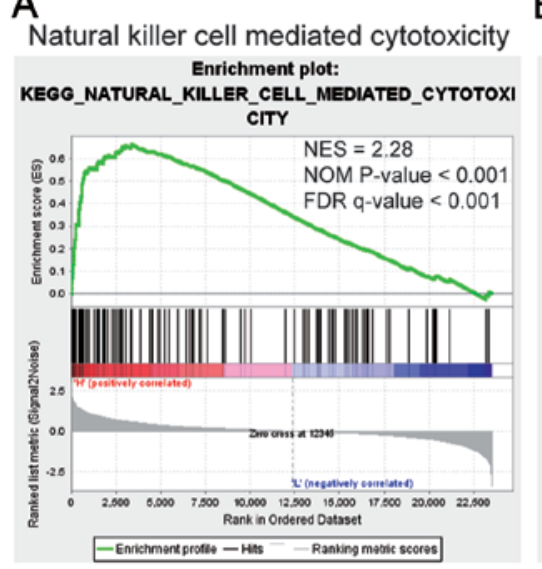

$D_{\text {Cytokine-cytokine receptor interaction }}$

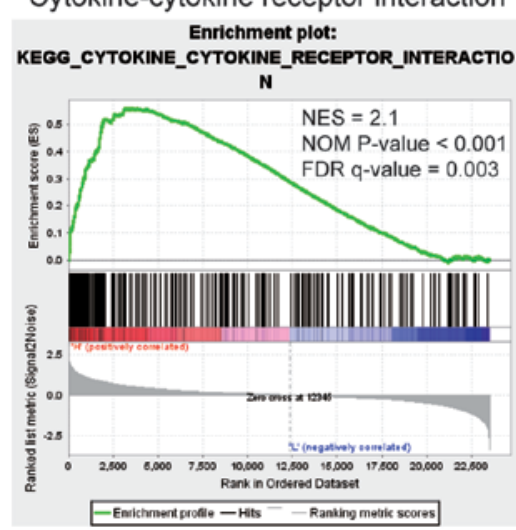

B

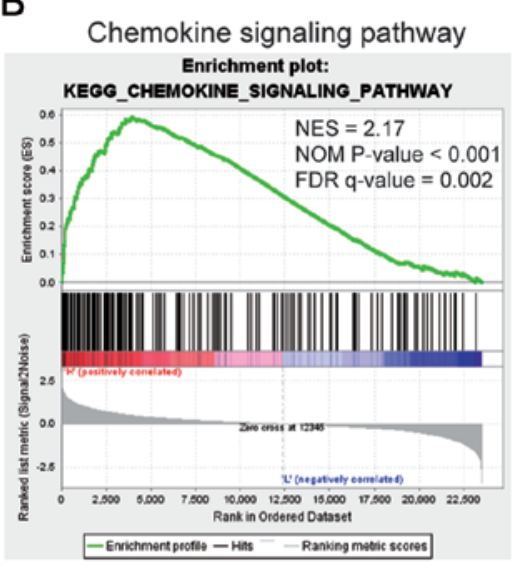

E

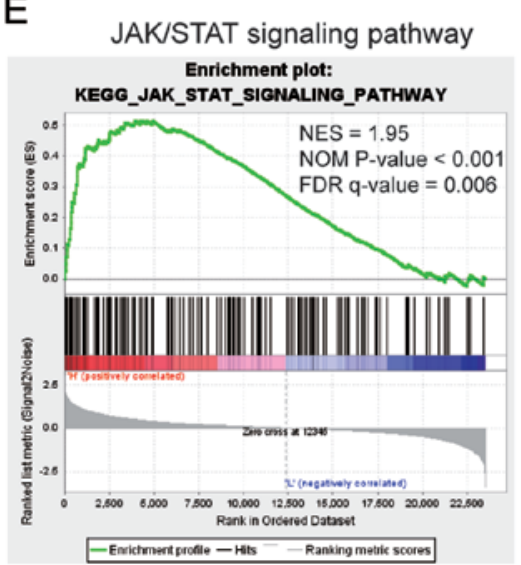

C

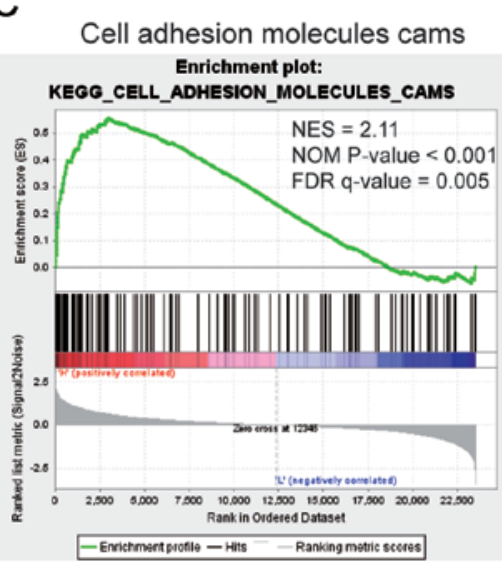

$\mathrm{F}$

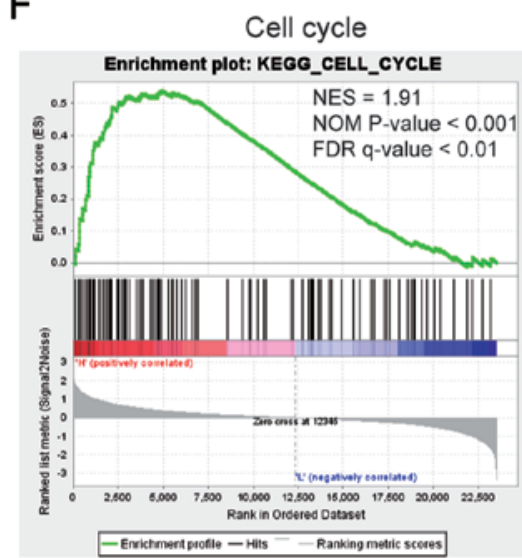

Figure 7. KEGG pathways associated with high CEP55 expression. (A) Natural killer cell-mediated cytotoxicity, (B) chemokine signaling pathway, (C) cell adhesion molecules CAMs, (D) cytokine-cytokine receptor interaction, (E) JAK/STAT signaling pathway and (F) cell cycle. CEP55, centrosomal protein 55; FDR, false discovery rate; KEGG, Kyoto Encyclopedia of Genes and Genomes; NES, normal enrichment score; NOM, normal.

marker to predict the diagnosis and prognosis of ccRCC. To understand the mechanism underlying the effects of CEP55 on tumorigenesis, the present study performed KEGG analysis using GSEA. CEP55 was significantly enriched in pathways associated with immunization, cell adhesion, inflammation, JAK/STAT signaling and cell proliferation. A recent study demonstrated that CEP55 promotes HCC cell migration and invasion via physiological interactions with JAK2 and by regulating the JAK2/STAT3/matrix metalloproteinase signaling pathway (37). Combined with the GSEA analysis, it was hypothesized that CEP55 may regulate cell proliferation and metastasis via the JAK/STAT signaling pathway.

In conclusion, the present study demonstrated that CEP55 may act as an oncogene that is highly expressed in ccRCC samples and associated with poor clinical outcomes; it may also be considered a diagnostic and prognostic biomarker for ccRCC. In addition, the results revealed that CEP55 may be associated with JAK2/STAT3 signaling pathway in tumor growth and metastasis. The present study has some limitations, as it was mainly based on public databases, and lacks in vivo and in vitro experiments. Tissue expression levels of CEP55, which can distinguish ccRCC patients from normal patients, only provides the potential diagnostic value of CEP55 in ccRCC and cannot be put into clinical application. Therefore, further study for the early detection of
CEP55 in blood samples from patients with ccRCC should be implemented, and the function and mechanism of CEP55 in ccRCC should be verified in cell and animal experiments.

\section{Acknowledgements}

The authors would like to thank Dr Yichen Chen (Ningbo Institution of Medicine and Science, Ningbo, China) for his technical support.

\section{Funding}

The present study was supported by The Natural Science Foundation of Ningbo (grant no. 2017A610186) and Medicine and Health Project of Zhejiang Province (grant no. 2019KY603).

\section{Availability of data and materials}

The datasets used and/or analyzed during the current study are available from the corresponding author on reasonable request.

\section{Authors' contributions}

LZ, ShiL and HL participated in the study design. XL and SheL collected the ccRCC samples and performed the 
RT-PCR analysis. LZ performed the experiments. LZ, ShiL, XL, MY, SheL and HL analyzed the data. LZ wrote the paper and submitted the manuscript, and ShiL, XL, MY and HL revised the manuscript. All authors read and approved the final manuscript.

\section{Ethics approval and consent to participate}

Ethics approval was obtained from the Ethics Committee of Ningbo Medical Centre Lihuili Hospital and written informed consent was obtained from all of the patients recruited.

\section{Patient consent for publication}

Informed consent from all participants was obtained for the publication of the present study.

\section{Competing interests}

The authors declare that they have no competing interests.

\section{References}

1. Ljungberg B, Campbell SC, Choi HY, Jacqmin D, Lee JE, Weikert S and Kiemeney LA: The epidemiology of renal cell carcinoma. Eur Urol 60: 615-621, 2011.

2. Znaor A, Lortet-Tieulent J, Laversanne M, Jemal A and Bray F: International variations and trends in renal cell carcinoma incidence and mortality. Eur Urol 67: 519-530, 2015.

3. Eikrem OS, Strauss P, Beisland C, Scherer A, Landolt L, Flatberg A, Leh S, Beisvag V, Skogstrand T, Hjelle K, et al: Development and confirmation of potential gene classifiers of human clear cell renal cell carcinoma using next-generation RNA sequencing. Scand J Urol 50: 452-462, 2016.

4. Jonasch E, Gao J and Rathmell WK: Renal cell carcinoma. BMJ 349: g4797, 2014.

5. Zhou L, Yin B, Liu Y, Hong Y, Zhang C and Fan J: Mechanism and function of decreased FOXO1 in renal cell carcinoma. J Surg Oncol 105: 841-847, 2012.

6. Ficarra V and Novara G: Kidney cancer: Characterizing late recurrence of renal cell carcinoma. Nat Rev Urol 10: 687-689, 2013.

7. Cancer Genome Atlas Research Network: Comprehensive molecular characterization of clear cell renal cell carcinoma. Nature 499: 43-49, 2013.

8. Ricketts CJ, De Cubas AA, Fan H, Smith CC, Lang M, Reznik E, Bowlby R, Gibb EA, Akbani R, Beroukhim R, et al: The cancer genome atlas comprehensive molecular characterization of renal cell carcinoma. Cell Rep 23: 3698, 2018.

9. Barrett T, Troup DB, Wilhite SE, Ledoux P, Evangelista C, Kim IF, Tomashevsky M, Marshall KA, Phillippy KH, Sherman PM, et al: NCBI GEO: Archive for functional genomics data sets-10 years on. Nucleic Acids Res 39 (Database Issue): D1005-D1010, 2011.

10. Cerami E, Gao J, Dogrusoz U, Gross BE, Sumer SO, Aksoy BA, Jacobsen A, Byrne CJ, Heuer ML, Larsson E, et al: The cBio cancer genomics portal: An open platform for exploring multidimensional cancer genomics data. Cancer Discov 2: 401-404, 2012.

11. Gao J, Aksoy BA, Dogrusoz U, Dresdner G, Gross B, Sumer SO, Sun Y, Jacobsen A, Sinha R, Larsson E, et al: Integrative analysis of complex cancer genomics and clinical profiles using the cBioPortal. Sci Signal 6: pl1, 2013.

12. Hanahan D and Weinberg RA: Hallmarks of cancer: The next generation. Cell 144: 646-674, 2011.

13. Edge SB, Byrd DR and Compton CC: AJCC cancer staging manual. 7th edition. Springer-Verlag, New York, pp547-560, 2009.

14. Eckel-Passow JE, Serie DJ, Bot BM, Joseph RW, Cheville JC and Parker AS: ANKS1B is a smoking-related molecular alteration in clear cell renal cell carcinoma. BMC Urol 14: 14, 2014.
15. Peña-Llopis S, Vega-Rubin-de-Celis S, Liao A, Leng $\mathrm{N}$, Pavía-Jiménez A, Wang S, Yamasaki T, Zhrebker L, Sivanand S, Spence P, et al: BAP1 loss defines a new class of renal cell carcinoma. Nat Genet 44: 751-759, 2012.

16. Gerlinger M, Horswell S, Larkin J, Rowan AJ, Salm MP, Varela I, Fisher R, McGranahan N, Matthews N, Santos CR, et al: Genomic architecture and evolution of clear cell renal cell carcinomas defined by multiregion sequencing. Nat Genet 46: 225-233, 2014

17. von Roemeling CA, Radisky DC, Marlow LA, Cooper SJ, Grebe SK, Anastasiadis PZ, Tun HW and Copland JA: Neuronal pentraxin 2 supports clear cell renal cell carcinoma by activating the AMPA-selective glutamate receptor-4. Cancer Res 74: 4796-4810, 2014

18. Ritchie ME, Phipson B, Wu D, Hu Y, Law CW, Shi W and Smyth GK: Limma powers differential expression analyses for RNA-sequencing and microarray studies. Nucleic Acids Res 43: e47, 2015

19. Kolde R, Laur S, Adler P and Vilo J: Robust rank aggregation for gene list integration and meta-analysis. Bioinformatics 28 : 573-580, 2012

20. Vosa U, Kolde R, Vilo J, Metspalu A and Annilo T: Comprehensive meta-analysis of microRNA expression using a robust rank aggregation approach. Methods Mol Biol 1182: 361-373, 2014.

21. Yang X, Zhu S, Li L, Zhang L, Xian S, Wang Y and Cheng Y: Identification of differentially expressed genes and signaling pathways in ovarian cancer by integrated bioinformatics analysis. Onco Targets Ther 11: 1457-1474, 2018.

22. Chen JS, Hung WS, Chan HH, Tsai SJ and Sun HS: In silico identification of oncogenic potential of fyn-related kinase in hepatocellular carcinoma. Bioinformatics 29: 420-427, 2013.

23. Livak KJ and Schmittgen TD: Analysis of relative gene expression data using real-time quantitative PCR and the 2(-Delta Delta C(T)) method. Methods 25: 402-408, 2001.

24. Metz CE: Basic principles of ROC analysis. Semin Nucl Med 8: 283-298, 1978.

25. Mootha VK, Lindgren CM, Eriksson KF, Subramanian A, Sihag S, Lehar J, Puigserver P, Carlsson E, Ridderstråle M, Laurila E, et al: PGC-1alpha-responsive genes involved in oxidative phosphorylation are coordinately downregulated in human diabetes. Nat Genet 34: 267-273, 2003.

26. Subramanian A, Tamayo P, Mootha VK, Mukherjee S, Ebert BL, Gillette MA, Paulovich A, Pomeroy SL, Golub TR, Lander ES and Mesirov JP: Gene set enrichment analysis: A knowledge-based approach for interpreting genome-wide expression profiles. Proc Natl Acad Sci USA 102: 15545-15550, 2005.

27. Takata M, Yamanaka N, Tanaka T, Yamanaka J, Maeda S, Okamoto E, Yasojima H, Uematsu K, Watanabe H and Uragari Y: What patients can survive disease free after complete resection for hepatocellular carcinoma? A multivariate analysis. Jpn J Clin Oncol 30: 75-81, 2000.

28. Girgis H,MasuiO,White NM,Scorilas A,RotondoF,Seivwright A, Gabril M, Filter ER, Girgis AH, Bjarnason GA, et al: Lactate dehydrogenase A is a potential prognostic marker in clear cell renal cell carcinoma. Mol Cancer 13: 101, 2014.

29. King SC, Pollack LA, Li J, King JB and Master VA: Continued increase in incidence of renal cell carcinoma, especially in young patients and high grade disease: United States 2001 to 2010. J Urol 191: 1665-1670, 2014.

30. Levi F, Ferlay J, Galeone C, Lucchini F, Negri E, Boyle P and La Vecchia C: The changing pattern of kidney cancer incidence and mortality in Europe. BJU Int 101: 949-958, 2008.

31. Jeffery J, Sinha D, Srihari S, Kalimutho M and Khanna KK: Beyond cytokinesis: The emerging roles of CEP55 in tumorigenesis. Oncogene 35: 683-690, 2016.

32. Zhao WM, Seki A and Fang G: Cep55, a microtubule-bundling protein, associates with centralspindlin to control the midbody integrity and cell abscission during cytokinesis. Mol Biol Cell 17: 3881-3896, 2006.

33. Fabbro M, Zhou BB, Takahashi M, Sarcevic B, Lal P, Graham ME, Gabrielli BG, Robinson PJ, Nigg EA, Ono Y and Khanna KK: Cdk1/Erk2- and Plk1-dependent phosphorylation of a centrosome protein, Cep55, is required for its recruitment to midbody and cytokinesis. Dev Cell 9: 477-488, 2005.

34. Jeffery J, Neyt C, Moore W, Paterson S, Bower NI, Chenevix-Trench G, Verkade H, Hogan BM and Khanna KK: Cep55 regulates embryonic growth and development by promoting Akt stability in zebrafish. FASEB J 29: 1999-2009, 2015. 
35. Xu ZY, Ma XS, Qi ST, Wang ZB, Guo L, Schatten H, Sun QY and Sun YP: Cep55 regulates spindle organization and cell cycle progression in meiotic oocyte. Sci Rep 5: 16978, 2015.

36. Chen $\mathrm{CH}, \mathrm{Lu} \mathrm{PJ}$, Chen YC, Fu SL, Wu KJ, Tsou AP, Lee YC, Lin TC, Hsu SL, Lin WJ, et al: FLJ10540-elicited cell transformation is through the activation of PI3-kinase/AKT pathway. Oncogene 26: 4272-4283, 2007.

37. Li M, Gao J, Li D and Yin Y: CEP55 promotes cell motility via JAK2-STAT3-MMPs cascade in hepatocellular carcinoma. Cells 7: pii: E99, 2018.

38. Yang YF, Zhang MF, Tian QH, Fu J, Yang X, Zhang CZ and Yang H: SPAG5 interacts with CEP55 and exerts oncogenic activities via PI3K/AKT pathway in hepatocellular carcinoma. Mol Cancer 17: 117, 2018

39. Sakai M, Shimokawa T, Kobayashi T, Matsushima S, Yamada Y Nakamura Y and Furukawa Y: Elevated expression of C10orf3 (chromosome 10 open reading frame 3 ) is involved in the growth of human colon tumor. Oncogene 25: 480-486, 2006.

40. Inoda S, Hirohashi Y, Torigoe T, Nakatsugawa M, Kiriyama K, Nakazawa E, Harada K, Takasu H, Tamura Y, Kamiguchi K, et al: Cep55/c10orf3, a tumor antigen derived from a centrosome residing protein in breast carcinoma. J Immunother 32: 474-485, 2009.

41. Wang Y, Jin T, Dai X and Xu J: Lentivirus-mediated knockdown of CEP55 suppresses cell proliferation of breast cancer cells Biosci Trends 10: 67-73, 2016

42. KalimuthoM,Sinha D, Jeffery J,Nones K, Srihari S, Fernando WC, Duijf PH, Vennin C, Raninga P, Nanayakkara D, et al: CEP55 is a determinant of cell fate during perturbed mitosis in breast cancer. EMBO Mol Med 10: pii: e8566, 2018.

43. Chen CH, Lai JM, Chou TY, Chen CY, Su LJ, Lee YC, Cheng TS, Hong YR, Chou CK, Whang-Peng J, et al: VEGFA upregulates FLJ10540 and modulates migration and invasion of lung cancer via PI3K/AKT pathway. PLoS One 4: e5052, 2009.

44. Liu L, Mei Q, Zhao J, Dai Y and Fu Q: Suppression of CEP55 reduces cell viability and induces apoptosis in human lung cancer. Oncol Rep 36: 1939-1945, 2016.

45. Wang G, Liu M, Wang H, Yu S, Jiang Z, Sun J, Han K, Shen J, Zhu M, Lin Z, et al: Centrosomal protein of 55 regulates glucose metabolism, proliferation and apoptosis of glioma cells via the Akt/mTOR signaling pathway. J Cancer 7: 1431-1440, 2016.

46. Zhu H, Chen D, Tang J, Huang C, Lv S, Wang D and Li G: Overexpression of centrosomal protein 55 regulates the proliferation of glioma cell and mediates proliferation promoted by EGFRvIII in glioblastoma U251 cells. Oncol Lett 15: 2700-2706, 2018.

47. Li F, Jin D, Tang C and Gao D: CEP55 promotes cell proliferation and inhibits apoptosis via the PI3K/Akt/p21 signaling pathway in human glioma U251 cells. Oncol Lett 15: 4789-4796, 2018.

48. Chen CH, Shiu LY, Su LJ, Huang CY, Huang SC, Huang CC, Yin YF, Wang WS, Tsai HT, Fang FM, et al: FLJ10540 is associated with tumor progression in nasopharyngeal carcinomas and contributes to nasopharyngeal cell proliferation, and metastasis via osteopontin/CD44 pathway. J Transl Med 10: 93, 2012.
49. Chen $\mathrm{CH}$, Chien $\mathrm{CY}$, Huang $\mathrm{CC}$, Hwang $\mathrm{CF}$, Chuang $\mathrm{HC}$, Fang FM, Huang HY, Chen CM, Liu HL and Huang CY: Expression of FLJ10540 is correlated with aggressiveness of oral cavity squamous cell carcinoma by stimulating cell migration and invasion through increased FOXM1 and MMP-2 activity. Oncogene 28: 2723-2737, 2009.

50. Xu L, Xia C, Sheng F, Sun Q, Xiong J and Wang S: CEP55 promotes the proliferation and invasion of tumour cells via the AKT signalling pathway in osteosarcoma. Carcinogenesis 39: 623-631, 2018.

51. Chen CH, Chang AY, Li SH, Tsai HT, Shiu LY, Su LJ, Wang WL, Chiu TJ, Luo SD, Huang TL and Chien CY: Suppression of Aurora-A-FLJ10540 signaling axis prohibits the malignant state of head and neck cancer. Mol Cancer 14: 83, 2015.

52. Weinberger P, Ponny SR, Xu H, Bai S, Smallridge R, Copland J and Sharma A: Cell cycle M-phase genes are highly upregulated in anaplastic thyroid carcinoma. Thyroid 27: 236-252, 2017.

53. Qi J, Liu G and Wang F: High levels of centrosomal protein 55 expression is associated with poor clinical prognosis in patients with cervical cancer. Oncol Lett 15: 9347-9352, 2018.

54. Jia Y, Xiao Z, Gongsun X, Xin Z, Shang B, Chen G, Wang Z and Jiang W: CEP55 promotes the proliferation, migration and invasion of esophageal squamous cell carcinoma via the PI3K/Akt pathway. Onco Targets Ther 11: 4221-4232, 2018.

55. Jiang W, Wang Z and Jia Y: CEP55 overexpression predicts poor prognosis in patients with locally advanced esophageal squamous cell carcinoma. Oncol Lett 13: 236-242, 2017.

56. Peng T, Zhou W, Guo F, Wu HS, Wang CY, Wang L and Yang ZY: Centrosomal protein 55 activates NF- $\mathrm{KB}$ signalling and promotes pancreatic cancer cells aggressiveness. Sci Rep 7: 5925, 2017.

57. Zheng H, Guo Z, Zheng X, Cheng $W$ and Huang $X$ : MicroRNA-144-3p inhibits cell proliferation and induces cell apoptosis in prostate cancer by targeting CEP55. Am J Transl Res 10: 2457-2468, 2018.

58. Kulkarni P and Uversky VN: Cancer/testis antigens: 'Smart' Biomarkers for diagnosis and prognosis of prostate and other cancers. Int J Mol Sci 18: pii: E740, 2017.

59. Jones J, Otu H, Spentzos D, Kolia S, Inan M, Beecken WD, Fellbaum C, Gu X, Joseph M, Pantuck AJ, et al: Gene signatures of progression and metastasis in renal cell cancer. Clin Cancer Res 11: 5730-5739, 2005.

60. Luo T, Chen X, Zeng S, Guan B, Hu B, Meng Y, Liu F, Wong T, $\mathrm{Lu}$ Y, Yun C, et al: Bioinformatic identification of key genes and analysis of prognostic values in clear cell renal cell carcinoma. Oncol Lett 16: 1747-1757, 2018.

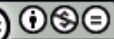

This work is licensed under a Creative Commons Attribution-NonCommercial-NoDerivatives 4.0 International (CC BY-NC-ND 4.0) License. 